\title{
Modifying SEIR models to explore the role of average household size in the era of lockdowns and social distancing
}

\author{
Paul Dempsey ${ }^{1}$ \\ ${ }^{1}$ Dazult, Maynooth, Co. Kildare, Ireland;
}

Email: paul.dempsey@dazult.com

\section{Abstract}

Compartmental models have long been used to study the dynamics of infectious diseases without requiring the intensive computational power of more detailed simulations. The well mixing assumption on which they are based is typically valid when people are meeting a significant number of other people each day. However, in the Covid-19 era of lockdowns and social distancing, the number of non-household contacts has dropped significantly. Standard SEIR models cannot produce the expected result of an idealised lockdown (i.e. no non-household contacts), where the final number of infected is less than the average household size times the number of infected and exposed at the time of the lockdown. To correct this anomaly, we separate the household and nonhousehold contributions to the total cases and apply a carrying capacity to the household acquired cases. Finally, we illustrate the application of the model to two countries with completely different approaches to managing the SARS-CoV-2 pandemic, New Zealand and Sweden. The sharp drop in cases in New Zealand following their lockdown can be well explained with a carrying capacity model, while we show that the Swedish approach could be extremely risky for countries with higher average household sizes. 


\section{Separating household and community effects in SEIR-type models}

The use of compartmental models in epidemiology, in particular SEIR-type (Susceptible, Exposed, Infectious, Removed), currently play an important tool in advising government policy through the SARS-CoV-2 pandemic [1]. There have been several attempts to estimate the impact of various social distancing measures on the effective reproduction number $\left(\mathrm{R}_{\mathrm{t}}\right)$, with stay at home advice ("lockdown") resulting in the largest percentage decrease [2]. The unique role of household transmission, and its violation of the well mixing assumption, appears to have been largely ignored. While in normal circumstances, where the number of non-household daily contacts exceeds the household contacts, ignoring this may be justified, it cannot be when the daily non-household contacts are similar to or less than the number of household contacts, as has been the case during since social distancing and lockdowns were introduced [3]. If complete data on household sizes is available, then rigorous methods are available to explore the impact of the breakdown of the well-mixing assumption [4]. In the absence of such data, average household sizes $\left(h_{s}\right)$ are typically available [5]. In what follows we crudely separate the household and community elements of the total cases, and, in the interest of brevity, focus only on the changes to the susceptible population $(S)$ as a result of the interactions with the infected population $(I)$. In compartmental models the change in $S$ can typically be described by

$$
\frac{d S}{d t}=-b k I S
$$

where $b$ is the average transmission rate between an infected individual and a susceptible one, and $k$ is the average number of contacts. Let us introduce two variables, $\mathrm{C}_{\mathrm{c}}$ and $\mathrm{C}_{\mathrm{h}}$, to represent the cases who have acquired the infection via the community and household, respectively. Then

$$
\frac{d C_{c}}{d t}=b_{c}\left(k-h_{s}+1\right) I S
$$

describes the rate of increase in community cases amongst the $\left(k-h_{s}+1\right)$ non-household daily contacts, where $b_{c}$ is the average transmission rate between an infected individual and a susceptible one in the community. The maximum number of household acquired infections in a population is $\left(h_{s}-1\right)$ times the number of community acquired infections. This can be used as a carrying capacity on the growth rate of household infections, 


$$
\frac{d C_{h}}{d t}=b_{h}\left(h_{s}-1\right) I S\left(1-\frac{C_{h}}{\left(h_{s}-1\right) C_{c}}\right)
$$

where $b_{h}$ is the average transmission rate between an infected individual and a susceptible one in a household setting.

The rate of change of $S$ can now be described by:

$$
\frac{d S}{d t}=-b_{c}\left(k-h_{s}+1\right) I S-b_{h}\left(h_{s}-1\right) I S\left(1-\frac{C_{h}}{\left(h_{s}-1\right) C_{c}}\right)
$$

with similar changes to the differential equations for the exposed $(E)$ or infectious $(I)$ compartments.

\section{Application to Covid-19 Data}

In the following applications we will use case and death data from the European Centre for Disease Prevention and Control (ECDC) [6] and an Erlang type SEIR model available at http://covid19.dazult.com/covid19.html . The model parameters used were $b_{c}=b_{h}=b=0.05249$ [7], a mean non-infectious latent period of 2.3 days (intervigintile range 0.41-5.46), a mean presymptomatic infectious period of 2.9 days $(0.52-6.88)$, and a further infectious period of 4.5 days (3.46-5.66) for both asymptomatic and symptomatic infected individuals. The asymptomatic fraction was set at $60 \%$. As only case and death data were used to estimate these values, they should only be taken as illustrative rather than definitive, but they are sufficient to demonstrate the impact of separating household from community spread in the era of social distancing and lockdowns. Country specific parameters include household size, number of non-household daily contacts, time to case notification, time to death, and case fatality rate.

\section{New Zealand}

New Zealand's average household size is 2.7 persons/household [5]. In the early stages of the pandemic the case data is well fitted by 12 non-household contacts for people without symptoms and 4 non-household contacts for people with symptoms. Following the announcement of an alert system on 21 March 2020 [8], the number of non-household contacts appear to drop to near zero (roughly 1 non-household contact per household every two-three days). Figure 1 shows the impact of the household carrying capacity model. The 
traditional model without household carrying capacity corresponds to people being in contact with 1.7 others in a well mixing environment. This cannot be set to a lower amount unless social distancing is achieved within a household. While other scenarios can be constructed, it is very hard to achieve the steep drop in cases during the New Zealand lockdown without the carrying capacity function.

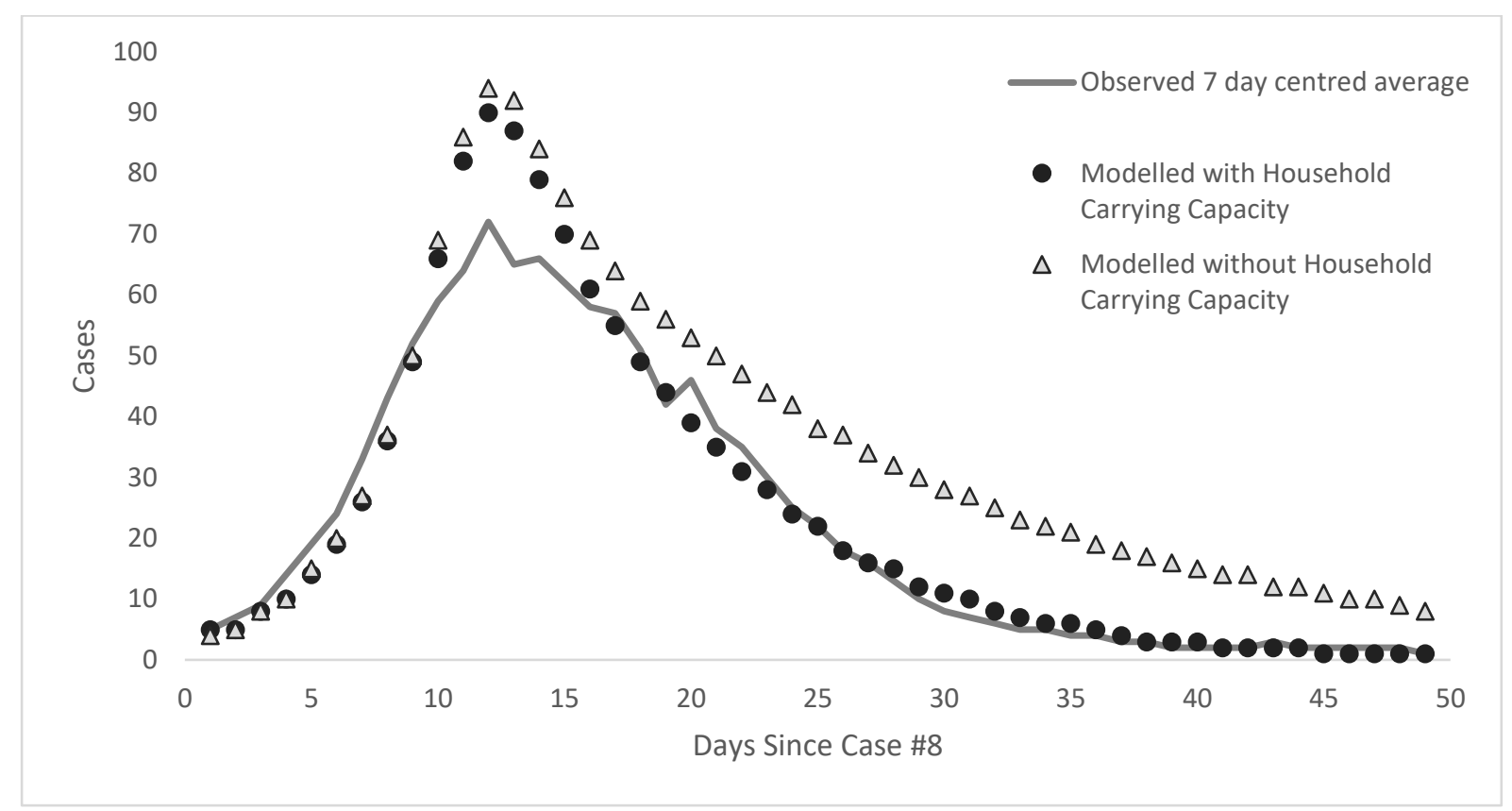

Fig. 1 Modelling the impact of New Zealand's lockdown on cases, with and without household carrying capacities

\section{Sweden}

Sweden's average household size is 2.2 persons/household [9]. Like New Zealand, in the early stages of the pandemic the case data is well fitted by 12 non-household contacts for people without symptoms and 6 non-household contacts for people with symptoms. Sweden's case data stopped being a useful metric around 12 March 2020 when a reduction in testing was announced [10], but it fatality data remains reliable. Unlike New Zealand, as Figure 2 shows, it is easy to construct parameters in the traditional SEIR model such that the results match those of the model with a household carrying capacity. However, if a country with a larger average household size was to try to follow Sweden's social distancing guidelines post their lockdown, they may experience a surge in cases, unless they have eliminated the virus. 


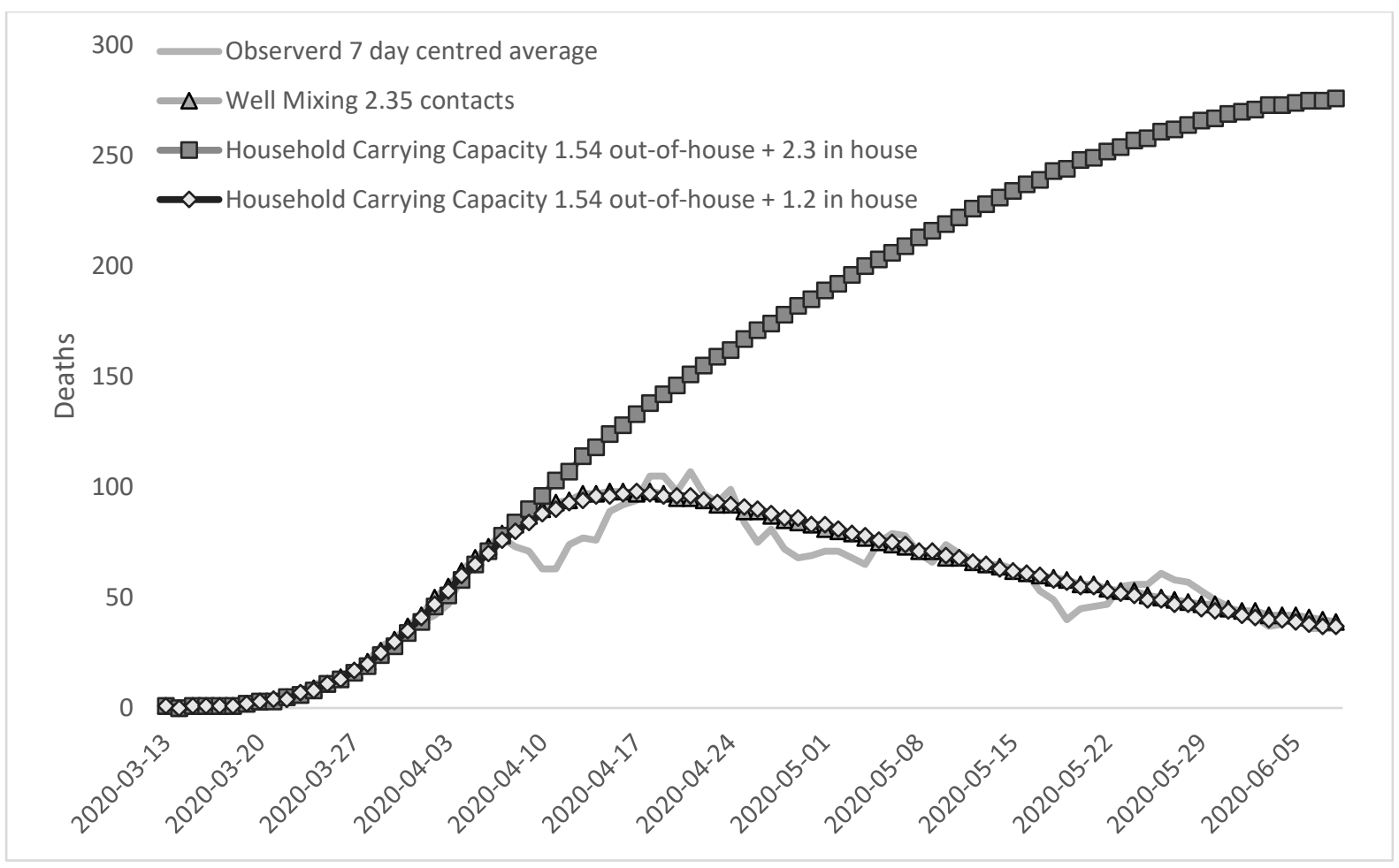

Fig. 2 Modelling the impact of Sweden's social distancing on deaths, with and without household carrying capacities

\section{References}

1. Irish Epidemiological Modelling Advisory Group Gov.ie - Irish Epidemiological Modelling Advisory Group to NPHET: Technical Notes. https://www.gov.ie/en/publication/dc5711irish-epidemiology-modelling-advisory-group-to-nphet-technical-notes/. Accessed 20 Jun 2020

2. Flaxman S, Mishra S, Gandy A, et al (2020) Estimating the effects of non-pharmaceutical interventions on COVID-19 in Europe. Nature 1-8

3. Del Fava E, Cimentada J, Perrotta D, Grow A, Rampazzo F, Gil-Clavel S, Zagheni E (2020) The differential impact of physical distancing strategies on social contacts relevant for the spread of COVID-19. medRxiv 2020.05.15.20102657

4. House T, Keeling MJ (2009) Household structure and infectious disease transmission. Epidemiol Infect 137:654-661

5. United Nations Household: Size and Composition 2018 - Countries. https://population.un.org/Household/index.html. Accessed 20 Jun 2020

6. European Centre for Disease Prevention and Control Download today's data on the geographic distribution of COVID-19 cases worldwide.

https://www.ecdc.europa.eu/en/publications-data/download-todays-data-geographicdistribution-covid-19-cases-worldwide. Accessed 20 Jun 2020

7. Yang Z, Zeng Z, Wang K, et al (2020) Modified SEIR and Al prediction of the epidemics trend of COVID-19 in China under public health interventions. J Thorac Dis 12:165-174 
8. Government of New Zealand Alert system overview | Unite for Recovery. https://uniteforrecovery.govt.nz/covid-19/covid-19-alert-system/alert-system-overview/. Accessed 20 Jun 2020

9. Esri MBRG Average Household Size in Sweden. https://www.arcgis.com/home/item.html?id=34e69daf9816437ab7429eb9ad7458f8. Accessed 20 Jun 2020

10. Edwards C Fact check: Has Sweden stopped testing people for the coronavirus? - The Local. https://www.thelocal.se/20200320/fact-check-has-sweden-stopped-testing-people-for-thecoronavirus. Accessed 20 Jun 2020

\section{Declarations}

\section{Funding}

Not applicable

\section{Conflicts of interest/Competing interests}

Not applicable

\section{Availability of data and material}

Daily Covid-19 case and death numbers are source from the European Centre for Disease Prevention and Control (ECDC) [6]

\section{Code availability}

Software and code used is available from http://covid19.dazult.com/covid19.html. 